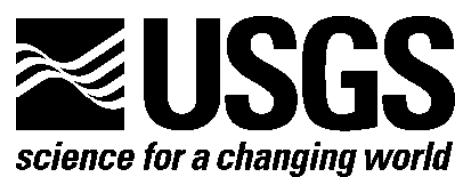

\title{
Variability of Distributions of Well-Scale Estimated Ultimate Recovery for Continuous (Unconventional) Oil and Gas Resources in the United States
}

By U.S. Geological Survey Oil and Gas Assessment Team

Open-File Report 2012-1118

U.S. Department of the Interior

U.S. Geological Survey 


\section{U.S. Department of the Interior \\ KEN SALAZAR, Secretary}

\section{U.S. Geological Survey \\ Marcia K. McNutt, Director}

U.S. Geological Survey, Reston, Virginia: 2012

For more information on the USGS-the Federal source for science about the Earth, its natural and living resources, natural hazards, and the environment-visit http://www.usgs.gov or call 1-888-ASK-USGS

For an overview of USGS information products, including maps, imagery, and publications, visit $h$ ttp://www.usgs.gov/pubprod

To order this and other USGS information products, visit $h$ ttp://store.usgs.gov

Suggested citation:

U.S. Geological Survey Oil and Gas Assessment Team, 2012, Variability of distributions of well-scale estimated ultimate recovery for continuous (unconventional) oil and gas resources in the United States: U.S. Geological Survey Open-File Report 2012-1118, 18 p.

Any use of trade, product, or firm names is for descriptive purposes only and does not imply endorsement by the U.S. Government.

Although this report is in the public domain, permission must be secured from the individual copyright owners to reproduce any copyrighted material contained within this report. 


\section{Contents}

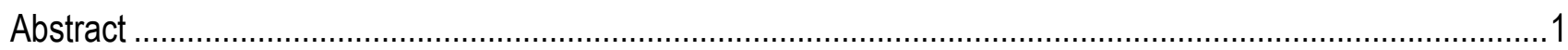

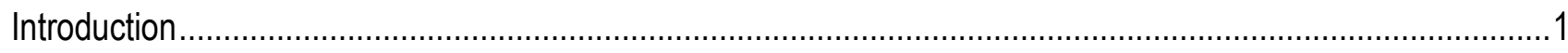

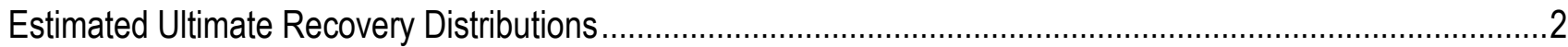

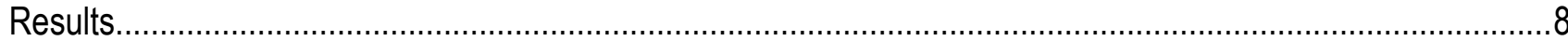

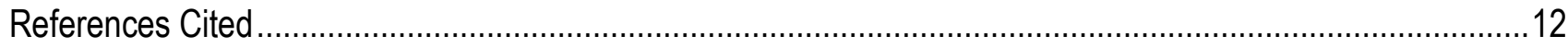

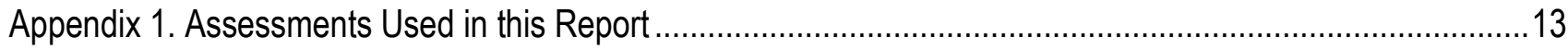

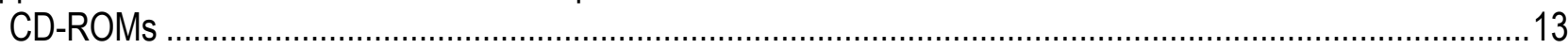

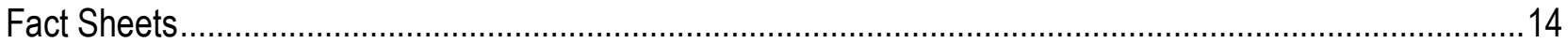

\section{Figures}

1. Cloud plot for United States shale-gas assessment units...................................................................

2. Cloud plot for United States coalbed-gas assessment units................................................................

3. Cloud plot for United States tight-gas assessment units..................................................................... 10

4. Cloud plot for United States continuous-oil assessment units. .............................................................11

\section{Tables}

1. Input data for estimated ultimate recovery distributions for United States shale-gas assessment units ..........3

2. Input data for estimated ultimate recovery distributions for United States coalbed-gas assessment units ......4

3. Input data for estimated ultimate recovery distributions for United States tight-gas assessment units ............5

4. Input data for estimated ultimate recovery distributions for United States continuous-oil assessment units 


\title{
Variability of Distributions of Well-Scale Estimated Ultimate Recovery for Continuous (Unconventional) Oil and Gas Resources in the United States
}

\author{
By U.S. Geological Survey Oil and Gas Assessment Team
}

\begin{abstract}
Since 2000, the U.S. Geological Survey has completed assessments of continuous (unconventional) resources in the United States based on geologic studies and analysis of wellproduction data. This publication uses those 132 continuous oil and gas assessments to show the variability of well productivity within and among the 132 areas. The production from the most productive wells in an area commonly is more than 100 times larger than that from the poorest productive wells. The 132 assessment units were classified into four categories: shale gas, coalbed gas, tight gas, and continuous oil. For each category, the mean well productivity in the most productive assessment units is considerably greater than that of the least productive assessment units.
\end{abstract}

\section{Introduction}

The U.S. Geological Survey (USGS) conducts quantitative assessments of potential oil and gas resources of the onshore United States and State waters. Since 2000, 132 assessments have been performed for continuous (unconventional) oil and gas resources, based on geologic studies and analysis of well-production data. Assessment methods are documented in Crovelli (2000, 2003), Klett and Charpentier (2003), Klett and Schmoker (2003), and Schmoker (2003). Each assessment unit (AU) was divided into cells, with each cell representing a well-drainage area. The estimates of resource potential were derived from estimates of the potential number of undrilled productive cells and of the productive capacities of those cells.

Estimated ultimate recovery (EUR) distributions were estimated for each AU, based on decline-curve analysis from monthly production data (IHS Energy, 2011) of hundreds to thousands of wells per AU. The EUR distribution used for each assessment calculation was specifically that for undrilled cells. Commonly, this EUR distribution for undrilled cells is closely similar to the distribution for drilled cells. In general, wells drilled early in the development of an AU, before drilling and completion techniques are optimized, have relatively low EURs. This can cause the estimated EURs for undrilled cells to be higher than those for drilled wells. Conversely, if the geologically most favorable parts of the AU have already been drilled, the EURs for undrilled cells may be lower than those of drilled wells.

The 132 AUs were classified into four categories: shale gas, coalbed gas, tight gas, and continuous oil. This categorization facilitated use of these data as analogs for hypothetical AUs. Sources for reports of these assessments are listed in appendix 1. 


\section{Estimated Ultimate Recovery Distributions}

Shifted truncated lognormal distributions were fit using the minimum, median, and maximum input values of estimated ultimate recovery (EUR). The upper end of the distribution was truncated at the 0.1 percent (1 in 1000) fractile.

$$
\begin{aligned}
& \mu=\ln \left(E U R_{\text {med }}-E U R_{\text {min }}\right) \\
& \sigma=\frac{\ln \left(\left(E U R_{\max }-E U R_{\min }\right) /\left(E U R_{\text {med }}-E U R_{\min }\right)\right)}{3.09}
\end{aligned}
$$

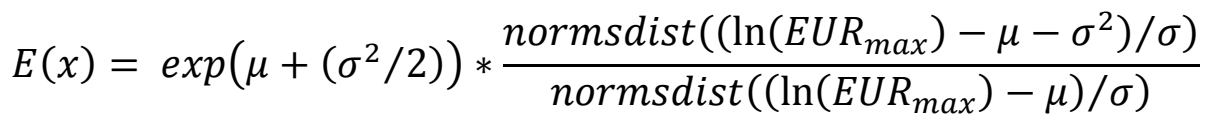

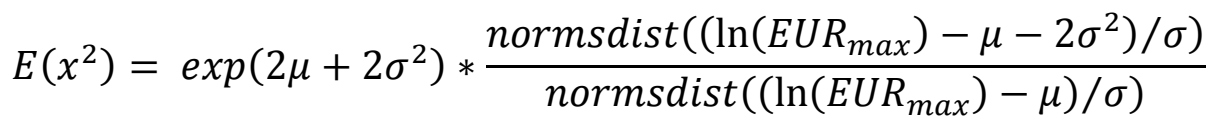

$$
\begin{aligned}
& E U R_{\text {mean }}=E U R_{\text {min }}+E(x) \\
& E U R_{s d}=\sqrt{E\left(x^{2}\right)-E(x)^{2}}
\end{aligned}
$$

where:

$$
\begin{aligned}
& E U R_{\text {min }}=\text { minimum EUR } \\
& E U R_{\text {med }}=\text { median EUR } \\
& E U R_{\max }=\text { maximum EUR } \\
& E U R_{\text {mean }}=\text { mean EUR } \\
& E U R_{s d}=\text { standard deviation of EUR } \\
& \text { normsdist }=\text { normal distribution function }
\end{aligned}
$$

The input values, as well as the calculated mean for each distribution, are given in tables 1 to 4 . 
Table 1. Input data for estimated ultimate recovery distributions for United States shale-gas assessment units, values in billions of cubic feet of natural gas. [AU, assessment unit; and EUR, estimated ultimate recovery]

\begin{tabular}{|c|c|c|c|c|c|c|c|}
\hline $\begin{array}{c}\text { AU } \\
\text { number }\end{array}$ & AU name & Province & $\begin{array}{c}\text { Year } \\
\text { assessed }\end{array}$ & $\begin{array}{c}\text { Minimum } \\
\text { EUR }\end{array}$ & $\begin{array}{l}\text { Median } \\
\text { EUR }\end{array}$ & $\begin{array}{c}\text { Maximum } \\
\text { EUR }\end{array}$ & $\begin{array}{c}\text { Mean } \\
\text { EUR }\end{array}$ \\
\hline 50490161 & Haynesville Sabine Platform Shale Gas & Gulf Coast Mesozoic & 2010 & 0.02 & 2 & 20 & 2.617 \\
\hline 50490163 & Mid-Bossier Sabine Platform Shale Gas & Gulf Coast Mesozoic & 2010 & 0.02 & 1 & 10 & 1.308 \\
\hline 50580161 & Woodford Shale Gas & Anadarko Basin & 2010 & 0.02 & 0.8 & 15 & 1.233 \\
\hline 50670468 & Interior Marcellus & Appalachian Basin & 2011 & 0.02 & 0.8 & 12 & 1.158 \\
\hline 50490167 & Eagle Ford Shale Gas & Gulf Coast Mesozoic & 2010 & 0.02 & 0.8 & 10 & 1.104 \\
\hline 50620362 & Fayetteville Shale Gas - High Gamma-Ray Depocenter & Arkoma Basin & 2010 & 0.02 & 0.8 & 10 & 1.104 \\
\hline 50450161 & Greater Newark East Frac-Barrier Continuous Barnett Shale Gas & Bend Arch-Fort Worth Basin & 2003 & 0.02 & 0.7 & 10 & 1.000 \\
\hline 50440161 & Delaware/Pecos Basins Woodford Continuous Shale Gas & Permian Basin & 2007 & 0.02 & 0.6 & 8 & 0.842 \\
\hline 50440162 & Delaware/Pecos Basins Barnett Continuous Shale Gas & Permian Basin & 2007 & 0.02 & 0.6 & 8 & 0.842 \\
\hline 50580261 & Thirteen Finger Limestone-Atoka Shale Gas & Anadarko Basin & 2010 & 0.02 & 0.5 & 10 & 0.785 \\
\hline 50620261 & Woodford Shale Gas & Arkoma Basin & 2010 & 0.02 & 0.5 & 10 & 0.785 \\
\hline 50210364 & Gothic, Chimney Rock, Hovenweep Shale Gas & Paradox Basin & 2011 & 0.02 & 0.4 & 10 & 0.672 \\
\hline 50630561 & Devonian Antrim Continuous Gas & Michigan Basin & 2004 & 0.02 & 0.4 & 4 & 0.523 \\
\hline 50620363 & Fayetteville Shale Gas - Western Arkansas Basin Margin & Arkoma Basin & 2010 & 0.02 & 0.3 & 6 & 0.470 \\
\hline 50210362 & Cane Creek Shale Gas & Paradox Basin & 2011 & 0.02 & 0.3 & 5 & 0.446 \\
\hline 50440163 & Midland Basin Woodford/Barnett Continuous Gas & Permian Basin & 2007 & 0.02 & 0.3 & 5 & 0.446 \\
\hline 50490165 & Maverick Basin Pearsall Shale Gas & Gulf Coast Mesozoic & 2010 & 0.02 & 0.25 & 5 & 0.391 \\
\hline 50450162 & Extended Continuous Barnett Shale Gas & Bend Arch-Fort Worth Basin & 2003 & 0.02 & 0.2 & 5 & 0.334 \\
\hline 50390761 & Niobrara Chalk & Denver Basin & 2001 & 0.025 & 0.2 & 2 & 0.261 \\
\hline 50620262 & Chattanooga Shale Gas & Arkoma Basin & 2010 & 0.02 & 0.1 & 6 & 0.223 \\
\hline 50670467 & Foldbelt Marcellus & Appalachian Basin & 2011 & 0.02 & 0.1 & 5 & 0.208 \\
\hline 50620364 & Caney Shale Gas & Arkoma Basin & 2010 & 0.02 & 0.08 & 5 & 0.179 \\
\hline 50670469 & Western Margin Marcellus & Appalachian Basin & 2011 & 0.02 & 0.05 & 5 & 0.129 \\
\hline 50640361 & Devonian to Mississippian New Albany Continuous Gas & Illinois Basin & 2007 & 0.01 & 0.08 & 1 & 0.110 \\
\hline 50670462 & Northwestern Ohio Shale & Appalachian Basin & 2002 & 0.01 & 0.04 & 0.5 & 0.055 \\
\hline 50670463 & Devonian Siltstone and Shale & Appalachian Basin & 2002 & 0.01 & 0.03 & 0.5 & 0.044 \\
\hline
\end{tabular}


Table 2. Input data for estimated ultimate recovery distributions for United States coalbed-gas assessment units, values in billions of cubic feet of natural gas. [AU, assessment unit; and EUR, estimated ultimate recovery]

\begin{tabular}{|c|c|c|c|c|c|c|c|}
\hline $\begin{array}{c}\mathrm{AU} \\
\text { number }\end{array}$ & AU name & Province & $\begin{array}{c}\text { Year } \\
\text { assessed }\end{array}$ & $\begin{array}{l}\text { Minimum } \\
\text { EUR }\end{array}$ & $\begin{array}{c}\text { Median } \\
\text { EUR }\end{array}$ & $\begin{array}{l}\text { Maximum } \\
\text { EUR }\end{array}$ & $\begin{array}{c}\text { Mean } \\
\text { EUR }\end{array}$ \\
\hline 50220181 & Fruitland Fairway Coalbed Gas & San Juan Basin & 2002 & 0.02 & 8 & 40 & 9.125 \\
\hline 50200181 & Northern Coal Fairway/Drunkards Wash & Uinta-Piceance & 2000 & 0.05 & 0.8 & 12 & 1.156 \\
\hline 50220182 & Basin Fruitland Coalbed Gas & San Juan Basin & 2002 & 0.02 & 0.6 & 20 & 1.110 \\
\hline 50200182 & Central Coal Fairway/Buzzards Bench & Uinta-Piceance & 2000 & 0.05 & 0.4 & 10 & 0.666 \\
\hline 50010181 & Nanushuk Formation Coalbed Gas & Northern Alaska & 2006 & 0.02 & 0.25 & 12 & 0.524 \\
\hline 50410182 & Vermejo Coalbed Gas & Raton Basin-Sierra Grande Uplift & 2004 & 0.02 & 0.25 & 9.5 & 0.481 \\
\hline 50200281 & Uinta Basin Blackhawk Coalbed Gas & Uinta-Piceance & 2000 & 0.05 & 0.25 & 10 & 0.480 \\
\hline 50360281 & Frontier-Adaville-Evanston Coalbed Gas & Wyoming Thrust Belt & 2003 & 0.02 & 0.4 & 2 & 0.456 \\
\hline 50410181 & Raton Coalbed Gas & Raton Basin-Sierra Grande Uplift & 2004 & 0.02 & 0.25 & 8 & 0.453 \\
\hline 50650281 & Warrior Basin & Warrior Basin & 2002 & 0.01 & 0.25 & 5 & 0.392 \\
\hline 50620481 & Arkoma Coalbed Gas & Arkoma Basin & 2010 & 0.02 & 0.3 & 3 & 0.392 \\
\hline 50330182 & Upper Fort Union Formation & Powder River Basin & 2000 & 0.02 & 0.23 & 4 & 0.345 \\
\hline 50200183 & Southern Coal Fairway & Uinta-Piceance & 2000 & 0.05 & 0.2 & 5 & 0.328 \\
\hline 50210581 & Kaiparowits Plateau & Paradox Basin & 2011 & 0.02 & 0.2 & 4 & 0.312 \\
\hline 50010183 & Sagavanirktok Formation Coalbed Gas & Northern Alaska & 2006 & 0.02 & 0.18 & 5 & 0.310 \\
\hline 50330181 & Wasatch Formation & Powder River Basin & 2000 & 0.02 & 0.18 & 3 & 0.267 \\
\hline 50370882 & Fort Union Coalbed Gas & Southwestern Wyoming & 2002 & 0.02 & 0.2 & 1.5 & 0.246 \\
\hline 50670581 & Pocahontas Basin & Appalachian Basin & 2002 & 0.01 & 0.15 & 2 & 0.210 \\
\hline 50350281 & Mesaverde Coalbed Gas & Wind River Basin & 2005 & 0.02 & 0.1 & 5 & 0.208 \\
\hline 50030281 & Cook Inlet Coalbed Gas & Southern Alaska & 2011 & 0.02 & 0.16 & 1.5 & 0.206 \\
\hline 50370881 & Lance Coalbed Gas & Southwestern Wyoming & 2002 & 0.02 & 0.15 & 1 & 0.180 \\
\hline 50200282 & Mesaverde Group Coalbed Gas & Uinta-Piceance & 2000 & 0.02 & 0.08 & 5 & 0.179 \\
\hline 50220381 & Menefee Coalbed Gas & San Juan Basin & 2002 & 0.02 & 0.08 & 5 & 0.179 \\
\hline 50200185 & Southern Coal Outcrop & Uinta-Piceance & 2001 & 0.05 & 0.1 & 3 & 0.165 \\
\hline 50670582 & Eastern Dunkard Basin & Appalachian Basin & 2002 & 0.01 & 0.1 & 2 & 0.156 \\
\hline 50040381 & Eocene Coalbed Gas & Western Oregon-Washington & 2009 & 0.02 & 0.1 & 2 & 0.155 \\
\hline 50010182 & Prince Creek-Tuluvak Formations Coalbed Gas & Northern Alaska & 2006 & 0.02 & 0.1 & 1.5 & 0.143 \\
\hline 50340281 & Mesaverde-Meeteetse Formation Coalbed Gas & Big Horn Basin & 2008 & 0.02 & 0.1 & 1.2 & 0.136 \\
\hline 50350282 & Meeteetse Coalbed Gas & Wind River Basin & 2005 & 0.02 & 0.08 & 2 & 0.131 \\
\hline 50350283 & Fort Union Coalbed Gas & Wind River Basin & 2005 & 0.02 & 0.08 & 2 & 0.131 \\
\hline 50370682 & Fort Union Coalbed Gas & Southwestern Wyoming & 2002 & 0.02 & 0.1 & 1 & 0.130 \\
\hline 50370981 & Wasatch-Green River Coalbed Gas & Southwestern Wyoming & 2002 & 0.02 & 0.1 & 0.8 & 0.124 \\
\hline 50311081 & Fort Union Coalbed Gas & Williston Basin & 2008 & 0.02 & 0.085 & 1 & 0.114 \\
\hline 50330183 & Lower Fort Union-Lance Formations & Powder River Basin & 2000 & 0.02 & 0.085 & 1 & 0.114 \\
\hline 50340282 & Fort Union Formation Coalbed Gas & Big Horn Basin & 2008 & 0.02 & 0.08 & 1 & 0.109 \\
\hline 50370581 & Mesaverde Coalbed Gas & Southwestern Wyoming & 2002 & 0.02 & 0.06 & 2 & 0.106 \\
\hline 50370681 & Mesaverde Coalbed Gas & Southwestern Wyoming & 2002 & 0.02 & 0.06 & 2 & 0.106 \\
\hline 50470381 & Wilcox Coalbed Gas & Western Gulf & 2007 & 0.01 & 0.05 & 0.5 & 0.065 \\
\hline 50640481 & Desmoinesian-Virgilian Coalbed Gas & Illinois Basin & 2007 & 0.01 & 0.03 & 0.25 & 0.037 \\
\hline 50470281 & Cretaceous Olmos Coalbed Gas & Western Gulf & 2007 & 0.01 & 0.03 & 0.1 & 0.032 \\
\hline
\end{tabular}


Table 3. Input data for estimated ultimate recovery distributions for United States tight-gas assessment units, values in billions of cubic feet of natural gas. [AU, assessment unit; and EUR, estimated ultimate recovery]

\begin{tabular}{|c|c|c|c|c|c|c|c|}
\hline $\begin{array}{c}\text { AU } \\
\text { number }\end{array}$ & AU name & Province & $\begin{array}{c}\text { Year } \\
\text { assessed }\end{array}$ & $\begin{array}{l}\text { Minimum } \\
\text { EUR }\end{array}$ & $\begin{array}{c}\text { Median } \\
\text { EUR }\end{array}$ & $\begin{array}{l}\text { Maximum } \\
\text { EUR }\end{array}$ & $\begin{array}{c}\text { Mean } \\
\text { EUR }\end{array}$ \\
\hline 50370661 & Mesaverde-Lance-Fort Union Continuous Gas & Southwestern Wyoming & 2002 & 0.02 & 1.2 & 15 & 1.657 \\
\hline 50370561 & Almond Continuous Gas & Southwestern Wyoming & 2002 & 0.02 & 0.9 & 20 & 1.460 \\
\hline 50200261 & Uinta Basin Continuous Gas & Uinta-Piceance & 2000 & 0.02 & 0.5 & 40 & 1.293 \\
\hline 50030161 & Tuxedni-Naknek Continuous Gas & Southern Alaska & 2011 & 0.02 & 0.6 & 30 & 1.286 \\
\hline 50620161 & Arkoma-Ouachita Foredeep Continuous & Arkoma Basin & 2010 & 0.02 & 0.6 & 30 & 1.286 \\
\hline 50350261 & Frontier-Muddy Continuous Gas & Wind River Basin & 2005 & 0.02 & 0.7 & 15 & 1.123 \\
\hline 50370261 & Mowry Continuous Gas & Southwestern Wyoming & 2002 & 0.02 & 0.7 & 15 & 1.123 \\
\hline 50350265 & Lance-Fort Union Sandstone Gas & Wind River Basin & 2005 & 0.02 & 0.6 & 20 & 1.110 \\
\hline 50370861 & Lance-Fort Union Continuous Gas & Southwestern Wyoming & 2002 & 0.02 & 0.8 & 10 & 1.104 \\
\hline 50370761 & Lewis Continuous Gas & Southwestern Wyoming & 2002 & 0.02 & 0.6 & 15 & 1.009 \\
\hline 50200362 & Uinta Basin Continuous Gas & Uinta-Piceance & 2000 & 0.02 & 0.5 & 16 & 0.911 \\
\hline 50200263 & Piceance Basin Continuous Gas & Uinta-Piceance & 2000 & 0.02 & 0.5 & 15 & 0.892 \\
\hline 50350264 & Mesaverde-Meeteetse Sandstone Gas & Wind River Basin & 2005 & 0.02 & 0.5 & 15 & 0.892 \\
\hline 50350262 & Cody Sandstones Continuous Gas & Wind River Basin & 2005 & 0.02 & 0.4 & 20 & 0.855 \\
\hline 50670364 & Tuscarora Basin Center & Appalachian Basin & 2002 & 0.01 & 0.7 & 4 & 0.817 \\
\hline 50220261 & Lewis Continuous Gas & San Juan Basin & 2002 & 0.02 & 0.5 & 6 & 0.683 \\
\hline 50220361 & Mesaverde Central-Basin Continuous Gas & San Juan Basin & 2002 & 0.02 & 0.5 & 6 & 0.683 \\
\hline 50220363 & Dakota-Greenhorn Continuous Gas & San Juan Basin & 2002 & 0.02 & 0.4 & 8 & 0.627 \\
\hline 50370461 & Hilliard-Baxter-Mancos Continuous Gas & Southwestern Wyoming & 2002 & 0.02 & 0.4 & 8 & 0.627 \\
\hline 50200161 & Deep (6,000 feet plus) Coal and Sandstone Gas & Uinta-Piceance & 2000 & 0.2 & 0.5 & 4 & 0.617 \\
\hline 50200262 & Uinta Basin Transitional Gas & Uinta-Piceance & 2000 & 0.02 & 0.25 & 15 & 0.570 \\
\hline 50340261 & Muddy-Frontier Sandstone and Mowry Fractured Shale Continuous Gas & Big Horn Basin & 2008 & 0.02 & 0.35 & 7.5 & 0.560 \\
\hline 50220362 & Mancos Sandstones Continuous Gas & San Juan Basin & 2002 & 0.02 & 0.35 & 5 & 0.499 \\
\hline 50370562 & Rock Springs-Ericson Continuous Gas & Southwestern Wyoming & 2002 & 0.02 & 0.4 & 3 & 0.491 \\
\hline 50200361 & Piceance Basin Continuous Gas & Uinta-Piceance & 2000 & 0.02 & 0.25 & 10 & 0.490 \\
\hline 50280163 & Eagle Sandstone and Claggett Shale West & North-Central Montana & 2000 & 0.01 & 0.25 & 9 & 0.475 \\
\hline 50220161 & Pictured Cliffs Continuous Gas & San Juan Basin & 2002 & 0.02 & 0.25 & 7 & 0.434 \\
\hline 50280162 & Eagle Sandstone and Claggett Shale East & North-Central Montana & 2000 & 0.01 & 0.2 & 7 & 0.375 \\
\hline
\end{tabular}


Table 3. Input data for estimated ultimate recovery distributions for United States tight-gas assessment units, values in billions of cubic feet of natural gas. [AU, assessment unit; and EUR, estimated ultimate recovery]-Continued

\begin{tabular}{|c|c|c|c|c|c|c|c|}
\hline $\begin{array}{c}\text { AU } \\
\text { number }\end{array}$ & AU name & Province & $\begin{array}{c}\text { Year } \\
\text { assessed }\end{array}$ & $\begin{array}{l}\text { Minimum } \\
\text { EUR }\end{array}$ & $\begin{array}{c}\text { Median } \\
\text { EUR }\end{array}$ & $\begin{array}{l}\text { Maximum } \\
\text { EUR }\end{array}$ & $\begin{array}{c}\text { Mean } \\
\text { EUR }\end{array}$ \\
\hline 50200363 & Uinta-Piceance Transitional and Migrated Gas & Uinta-Piceance & 2000 & 0.02 & 0.2 & 7 & 0.373 \\
\hline 50200264 & Piceance Basin Transitional Gas & Uinta-Piceance & 2000 & 0.02 & 0.25 & 4 & 0.367 \\
\hline 50280166 & Greenhorn-Upper Belle Fourche & North-Central Montana & 2000 & 0.01 & 0.2 & 6 & 0.356 \\
\hline 50280167 & Bowdoin Dome & North-Central Montana & 2000 & 0.01 & 0.2 & 5 & 0.336 \\
\hline 50340263 & Cody Sandstone Continuous Gas & Big Horn Basin & 2008 & 0.02 & 0.2 & 5 & 0.334 \\
\hline 50340264 & Mesaverde Sandstone Continuous Gas & Big Horn Basin & 2008 & 0.02 & 0.2 & 5 & 0.334 \\
\hline 50280165 & Greenhorn-Lower Belle Fourche & North-Central Montana & 2000 & 0.01 & 0.25 & 2.5 & 0.327 \\
\hline 50050161 & Columbia Basin Continuous Gas & $\begin{array}{l}\text { Eastern Oregon and } \\
\text { Washington }\end{array}$ & 2006 & 0.02 & 0.2 & 3 & 0.288 \\
\hline 50390662 & Dakota Group Basin-Center Gas & Denver Basin & 2001 & 0.02 & 0.2 & 2.5 & 0.275 \\
\hline 50670461 & Greater Big Sandy & Appalachian Basin & 2002 & 0.01 & 0.15 & 2 & 0.210 \\
\hline 50330461 & Shallow Continuous Biogenic Gas & Powder River Basin & 2002 & 0.01 & 0.08 & 1.5 & 0.122 \\
\hline 50670361 & Clinton-Medina Basin Center & Appalachian Basin & 2002 & 0.01 & 0.08 & 1.2 & 0.115 \\
\hline 50670465 & Catskill Sandstones and Siltstones & Appalachian Basin & 2002 & 0.01 & 0.07 & 1.5 & 0.111 \\
\hline 50280161 & Judith River Formation & North-Central Montana & 2000 & 0.01 & 0.06 & 2 & 0.109 \\
\hline 50280164 & Niobrara-Carlile & North-Central Montana & 2000 & 0.01 & 0.07 & 1 & 0.099 \\
\hline 50670363 & Clinton-Medina Transitional & Appalachian Basin & 2002 & 0.01 & 0.06 & 1 & 0.089 \\
\hline 50670362 & Clinton-Medina Transitional Northeast & Appalachian Basin & 2002 & 0.01 & 0.06 & 0.9 & 0.086 \\
\hline 50670466 & Berea Sandstone & Appalachian Basin & 2002 & 0.01 & 0.03 & 0.5 & 0.044 \\
\hline
\end{tabular}


Table 4. Input data for estimated ultimate recovery distributions for United States continuous-oil assessment units, values in millions of barrels of oil. [AU, assessment unit; and EUR, estimated ultimate recovery]

\begin{tabular}{|c|c|c|c|c|c|c|c|}
\hline $\begin{array}{c}\text { AU } \\
\text { number }\end{array}$ & AU name & Province & $\begin{array}{c}\text { Year } \\
\text { assessed }\end{array}$ & $\begin{array}{l}\text { Minimum } \\
\text { EUR }\end{array}$ & $\begin{array}{c}\text { Median } \\
\text { EUR }\end{array}$ & $\begin{array}{l}\text { Maximum } \\
\text { EUR }\end{array}$ & $\begin{array}{c}\text { Mean } \\
\text { EUR }\end{array}$ \\
\hline 50310164 & Eastern Expulsion Threshold & Williston Basin & 2008 & 0.002 & 0.12 & 5 & 0.241 \\
\hline 50310163 & Nesson-Little Knife Structural & Williston Basin & 2008 & 0.002 & 0.09 & 4 & 0.185 \\
\hline 50210361 & Cane Creek Shale Oil & Paradox Basin & 2011 & 0.002 & 0.08 & 3 & 0.154 \\
\hline 50310165 & Northwest Expulsion Threshold & Williston Basin & 2008 & 0.002 & 0.065 & 4 & 0.151 \\
\hline 50310161 & Elm Coulee-Billings Nose & Williston Basin & 2008 & 0.002 & 0.08 & 2 & 0.135 \\
\hline 50270561 & Marias River Shale Continuous Oil & Montana Thrust Belt & 2002 & 0.001 & 0.08 & 1.6 & 0.126 \\
\hline 50370361 & Niobrara Continuous Oil & Southwestern Wyoming & 2002 & 0.001 & 0.08 & 1.6 & 0.126 \\
\hline 50300361 & Niobrara Continuous Oil & Hanna, Laramie, Shirley Basins & 2005 & 0.001 & 0.04 & 1.6 & 0.079 \\
\hline 50310162 & Central Basin-Poplar Dome & Williston Basin & 2008 & 0.002 & 0.025 & 2 & 0.064 \\
\hline 50210363 & Gothic, Chimney Rock, Hovenweep Shale Oil & Paradox Basin & 2011 & 0.002 & 0.03 & 1.5 & 0.064 \\
\hline 50580162 & Woodford Shale Oil & Anadarko Basin & 2010 & 0.003 & 0.03 & 1.5 & 0.064 \\
\hline 50200561 & Deep Uinta Overpressured Continuous Oil & Uinta-Piceance & 2000 & 0.003 & 0.045 & 0.45 & 0.059 \\
\hline 50440165 & Spraberry Continuous Oil & Permian Basin & 2007 & 0.001 & 0.045 & 0.4 & 0.057 \\
\hline 50490170 & Eagle Ford Shale Oil & Gulf Coast Mesozoic & 2010 & 0.002 & 0.03 & 1 & 0.055 \\
\hline 50490168 & Austin Pearsall-Giddings Area Oil & Gulf Coast Mesozoic & 2010 & 0.002 & 0.04 & 0.5 & 0.055 \\
\hline 50330361 & Niobrara Continuous Oil & Powder River Basin & 2002 & 0.002 & 0.028 & 0.5 & 0.042 \\
\hline 50330261 & Mowry Continuous Oil & Powder River Basin & 2002 & 0.002 & 0.025 & 0.35 & 0.035 \\
\hline 50340262 & Mowry Fractured Shale Continuous Oil & Big Horn Basin & 2008 & 0.002 & 0.025 & 0.35 & 0.035 \\
\hline 50390261 & Fractured Niobrara Limestone (Silo Field Area) & Denver Basin & 2001 & 0.002 & 0.022 & 0.4 & 0.033 \\
\hline 50390661 & Niobrara-Codell (Wattenberg Area) & Denver Basin & 2001 & 0.003 & 0.008 & 0.1 & 0.011 \\
\hline
\end{tabular}




\section{Results}

The results are presented in figures 1 through 4 . Each line shows the range of EURs for a single AU. Only those EURs greater than the minimum assessed value (for that particular AU assessment) are included. Individual AU distributions show approximately two orders of magnitude difference between the smallest and largest EURs within a single AU. This range would be even larger if the distributions were not truncated.

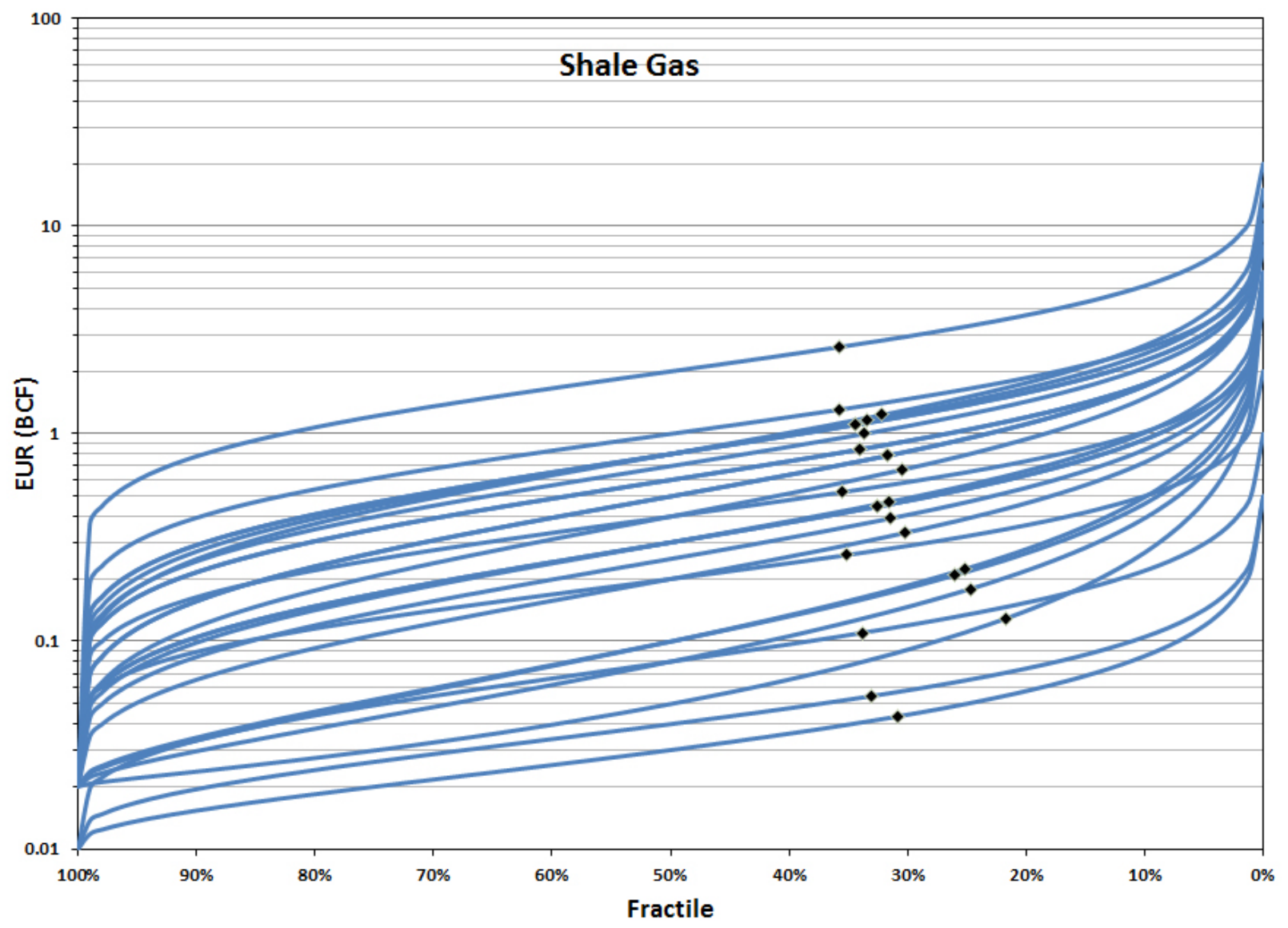

Figure 1. Cloud plot for United States shale-gas assessment units. Each curve represents one assessment unit and is based on the input data in table 1. Black diamonds indicate the mean value for each curve. [AU, assessment unit; EUR, estimated ultimate recovery; and BCF, billions of cubic feet] 


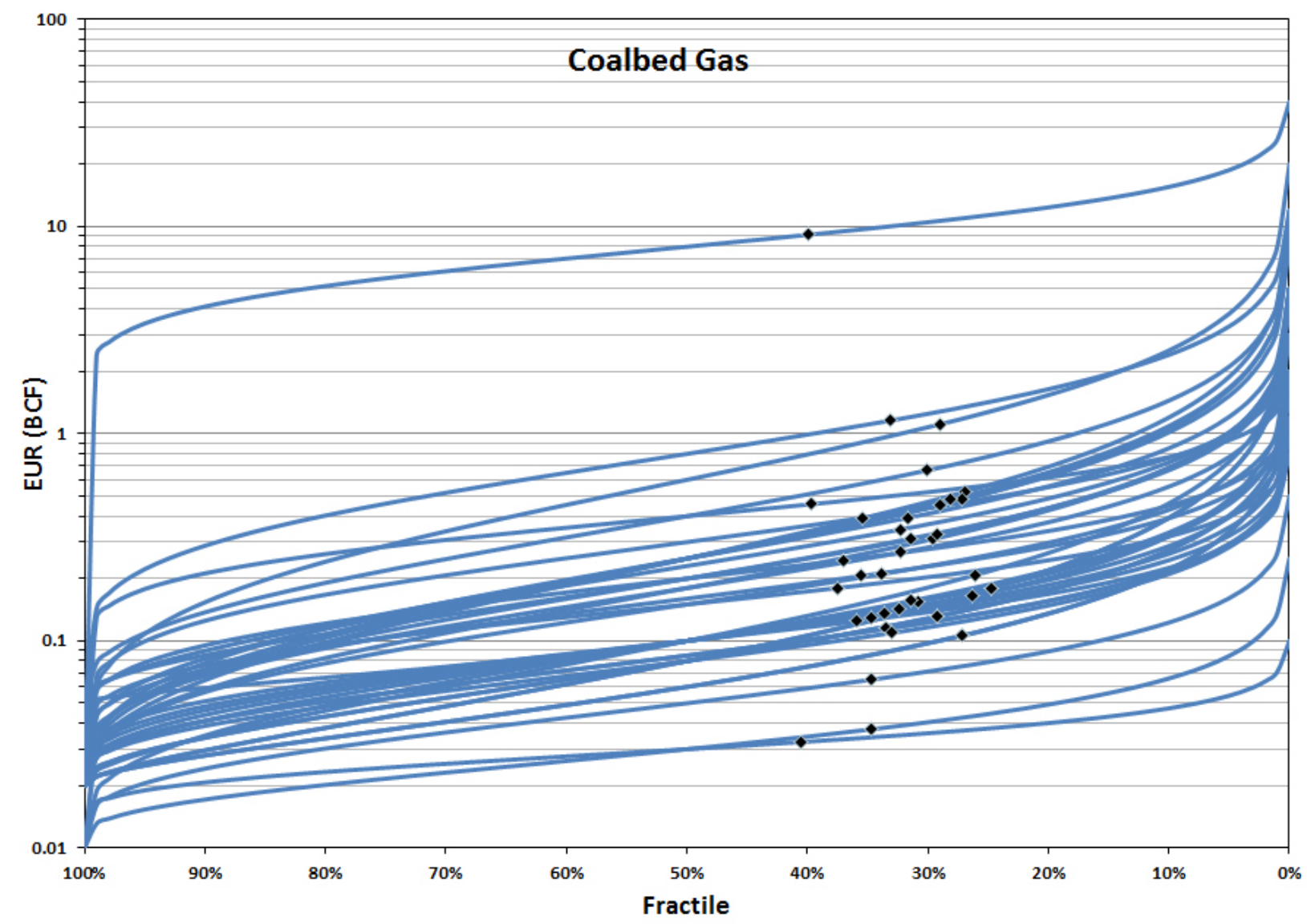

Figure 2. Cloud plot for United States coalbed-gas assessment units. Each curve represents one assessment unit and is based on the input data in table 2. Black diamonds indicate the mean value for each curve. [AU, assessment unit; EUR, estimated ultimate recovery; and BCF, billions of cubic feet] 


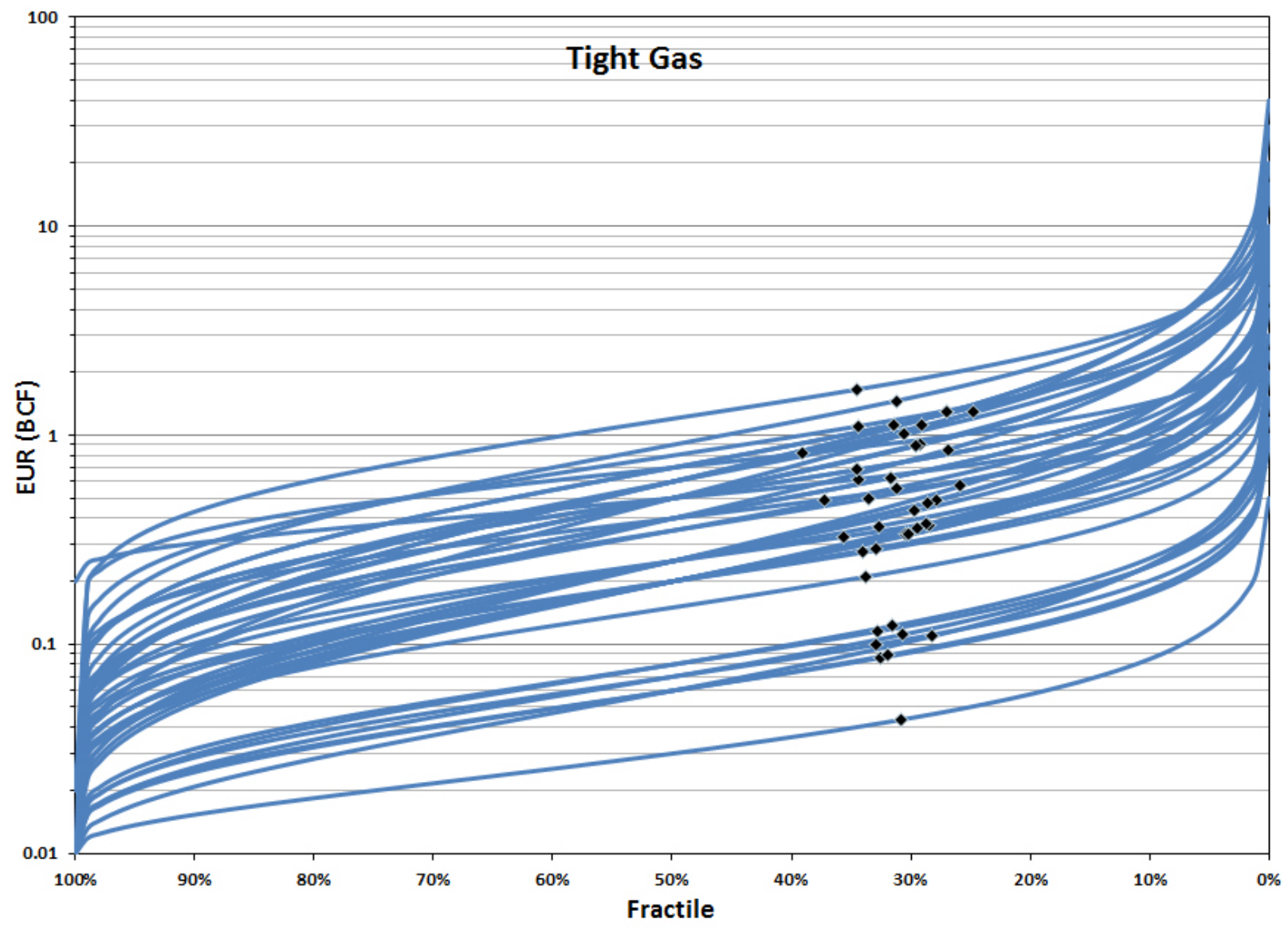

Figure 3. Cloud plot for United States tight-gas assessment units. Each curve represents one assessment unit and is based on the input data in table 3 . Black diamonds show the mean value for each curve. [AU, assessment unit; EUR, estimated ultimate recovery; and BCF, billions of cubic feet] 


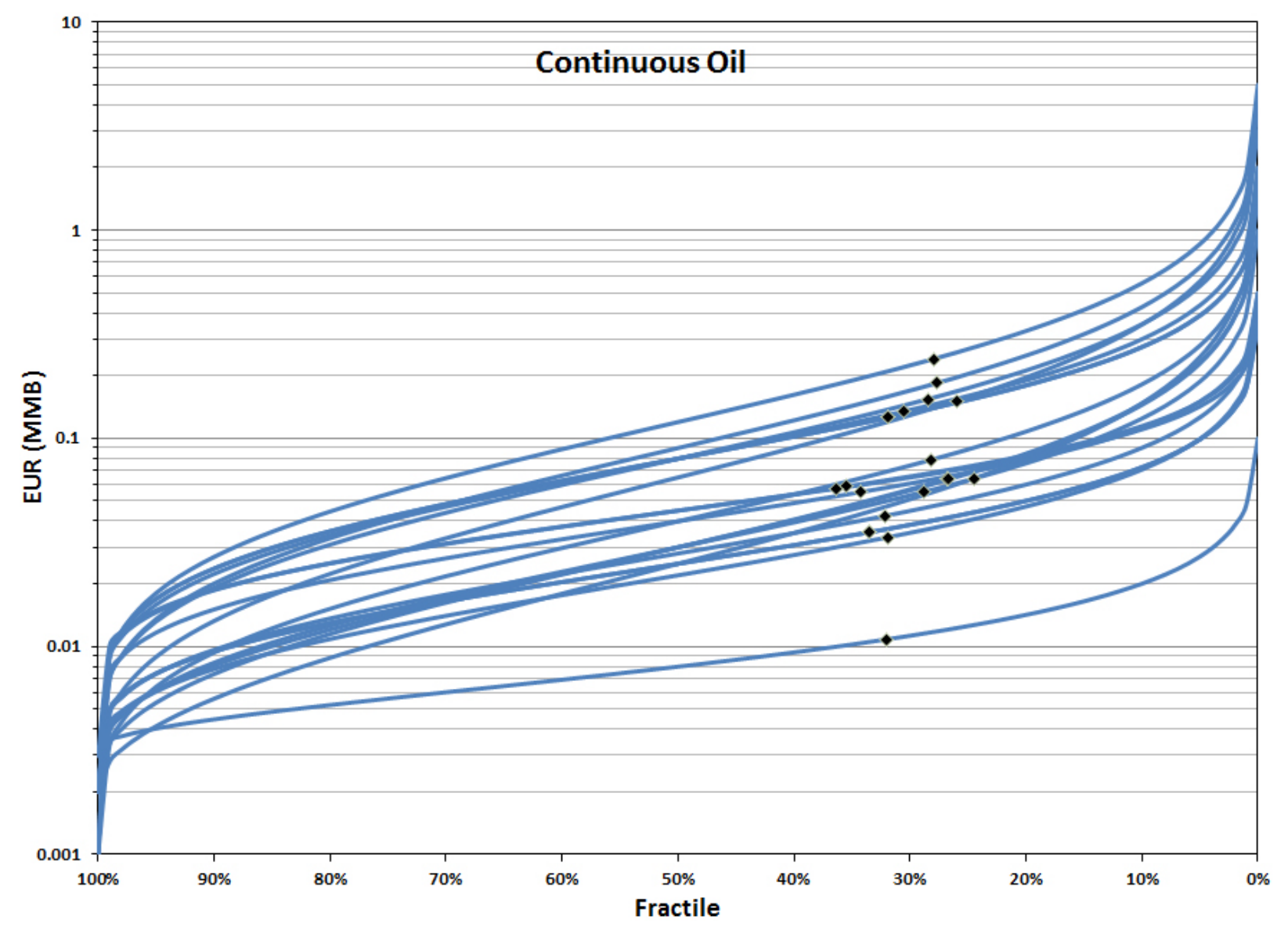

Figure 4. Cloud plot for United States continuous-oil assessment units. Each curve represents one assessment unit and is based on the input data in table 4. Black diamonds indicate the mean value for each curve. [AU, assessment unit; EUR, estimated ultimate recovery; and MMB, millions of barrels]

Each figure shows the EUR curves for a single category (shale gas, coalbed gas, tight gas, and continuous oil), allowing comparison of EUR distributions among AUs. The four figures are termed "cloud plots," which show the "cloud" of data representing the distribution of EUR distributions. Cloud plots of the distributions of drilled wells show similar ranges of variability.

Individual cloud plots show the wide variability among AUs of a particular category. The most productive AUs have average EURs from 22 to almost 300 times those of the least productive AUs. Also note the strong similarity of the shale gas and tight gas clouds (figs. 1,3). 


\section{References Cited}

Crovelli, R.A., 2000, Analytic resource assessment method for continuous (unconventional) oil and gas accumulations - the "ACCESS" method: U.S. Geological Survey Open-File Report 00-044, 34 p. (Also available at http://pubs.usgs.gov/of/2000/0044/report.pdf)

Crovelli, R.A., 2003, Analytic resource assessment method for continuous petroleum accumulations - the ACCESS assessment method, chap. 22, in U.S. Geological Survey UintaPiceance Assessment Team, Petroleum Systems and Geologic Assessment of Oil and Gas in the Uinta-Piceance Province, Utah and Colorado: U.S. Geological Survey Digital Data Series DDS-69-B, 10 p. (Also available at http://pubs.usgs.gov/dds/dds-069/dds-069-b/)

IHS Energy, 2011, U.S. Production and Well Data: Englewood, Colo., database available from IHS Energy, 15 Inverness Way East, D205, Englewood, CO 80112, U.S.A.

Klett, T.R., and Charpentier, R.R., 2003, FORSPAN model users guide: U.S. Geological Survey Open-File Report 03-354, 37 p. (Also available at http://pubs.usgs.gov/of/2003/ofr-03-354/)

Klett, T.R., and Schmoker, J.W., 2003, U.S. Geological Survey input-data form and operational procedure for the assessment of continuous petroleum accumulations, chap. 18, in U.S. Geological Survey Uinta-Piceance Assessment Team, Petroleum Systems and Geologic Assessment of Oil and Gas in the Uinta-Piceance Province, Utah and Colorado: U.S. Geological Survey Digital Data Series DDS-69-B, 8 p. (Also available at http://pubs.usgs.gov/dds/dds-069/dds-069-b/)

Schmoker, J.W., 2003, U.S. Geological Survey assessment concepts for continuous petroleum accumulations, chap. 17, in U.S. Geological Survey Uinta-Piceance Assessment Team, Petroleum Systems and Geologic Assessment of Oil and Gas in the Uinta-Piceance Province, Utah and Colorado: U.S. Geological Survey Digital Data Series DDS-69-B, 7 p. (Also available at http://pubs.usgs.gov/dds/dds-069/dds-069-b/) 


\section{Appendix 1. Assessments Used in this Report}

\section{CD-ROMs}

Higley, D.K., compiler, 2007, Petroleum systems and assessment of undiscovered oil and gas in the Raton Basin-Sierra Grande Uplift Province, Colorado and New Mexico-USGS Province 41: U.S. Geological Survey Digital Data Series DDS-69-N, 1 CD-ROM. (Available at http://pubs.usgs.gov/dds/dds-069/dds-069-n/)

Higley, D.K., compiler, 2007, Petroleum systems and assessment of undiscovered oil and gas in the Denver Basin Province, Colorado, Kansas, Nebraska, South Dakota, and WyomingUSGS Province 39: U.S. Geological Survey Digital Data Series DDS-69-P, 1 CD-ROM. (Available at http://pubs.usgs.gov/dds/dds-069/dds-069-p/)

Roberts, S.B., compiler, 2008, Geologic assessment of undiscovered, technically recoverable coalbed-gas resources in Cretaceous and Tertiary rocks, North Slope and adjacent State waters, Alaska: U.S. Geological Survey Digital Data Series DDS-69-S, 1 CD-ROM. (Available at http://pubs.usgs.gov/dds/dds-069/dds-069-s/)

U.S. Geological Survey Bighorn Basin Assessment Team, 2010, Petroleum systems and geologic assessment of oil and gas in the Bighorn Basin Province, Wyoming and Montana: U.S. Geological Survey Digital Data Series DDS-69-V, 1 CD-ROM. (Available at http://pubs.usgs.gov/dds/dds-069/dds-069-v/)

U.S. Geological Survey Black Warrior Basin Province Assessment Team, 2007, Geologic assessment of undiscovered oil and gas resources of the Black Warrior Basin Province, Alabama and Mississippi: U.S. Geological Survey Digital Data Series DDS-69-I, 1 CD-ROM. (Available at http://pubs.usgs.gov/dds/dds-069/dds-069-i/)

U.S. Geological Survey Eastern Oregon and Washington Province Assessment Team, 2008, Geologic assessment of undiscovered gas resources of the Eastern Oregon and Washington Province: U.S. Geological Survey Digital Data Series DDS-69-O, 1 CD-ROM. (Available at http://pubs.usgs.gov/dds/dds-069/dds-069-o/)

U.S. Geological Survey Hanna, Laramie, and Shirley Basins Province Assessment Team, 2007, Petroleum systems and geologic assessment of undiscovered oil and gas, Hanna, Laramie, and Shirley Basins Province, Wyoming and Colorado: U.S. Geological Survey Digital Data Series DDS-69-K, 1 CD-ROM. (Available at http://pubs.usgs.gov/dds/dds-069/dds-069-k/)

U.S. Geological Survey Powder River Basin Assessment Team, 2009, Total petroleum systems and geologic assessment of oil and gas resources in the Powder River Basin Province, Wyoming and Montana: U.S. Geological Survey Digital Data Series DDS-69-U, 1 CD-ROM (revised April 2010). (Available at http://pubs.usgs.gov/dds/dds-069/dds-069-u/)

U.S. Geological Survey Powder River Basin Province Assessment Team, 2004, Total petroleum system and assessment of coalbed gas in the Powder River Basin Province, Wyoming and 
Montana: U.S. Geological Survey Digital Data Series DDS-69-C, 1 CD-ROM. (Available at http://pubs.usgs.gov/dds/dds-069/dds-069-c/)

U.S. Geological Survey Southwestern Wyoming Province Assessment Team, 2005, Petroleum systems and geologic assessment of oil and gas in the Southwestern Wyoming Province, Wyoming, Colorado and Utah: U.S. Geological Survey Digital Data Series DDS-69-D, 1 CDROM. (Available at http://pubs.usgs.gov/dds/dds-069/dds-069-d/)

U.S. Geological Survey Western Oregon and Washington Province Assessment Team, 2011, Geologic assessment of undiscovered hydrocarbon resources of the Western Oregon and Washington Province: U.S. Geological Survey Digital Data Series DDS-69-X, 1 CD-ROM. (Available at $h t t p: / / p u b s . u s g s . g o v / d d s / d d s-069 / d d s-069-x /$ )

U.S. Geological Survey Williston Basin Province Assessment Team, 2011, Assessment of undiscovered oil and gas resources of the Williston Basin Province of North Dakota, Montana, and South Dakota, 2010: U.S. Geological Survey Digital Data Series DDS-69-W, 1 CDROM. (Available at http://pubs.usgs.gov/dds/dds-069/dds-069-w/)

USGS Uinta-Piceance Assessment Team, compilers, 2003, Petroleum systems and geologic assessment of oil and gas in the Uinta-Piceance Province, Utah and Colorado: U.S. Geological Survey Digital Data Series DDS-69-B, 1 CD-ROM. (Available at http://pubs.usgs.gov/dds/dds-069/dds-069-b/)

USGS Wind River Basin Province Assessment Team, 2007, Petroleum systems and geologic assessment of oil and gas in the Wind River Basin Province, Wyoming: U.S. Geological Survey Digital Data Series DDS-69-J, 1 CD-ROM. (Available at http://pubs.usgs.gov/dds/dds-069/dds-069-j/)

\section{Fact Sheets}

Anna, L.O., Charpentier, R.R., Cook, T.A., Klett, T.R., Pollastro, R.M., and Schenk, C.J., 2006, Assessment of undiscovered oil and gas resources of the Powder River Basin Province of Wyoming and Montana—2006 update: U.S. Geological Survey Fact Sheet 2006-3135, 2 p. (Available at $h t t p: / / p u b s . u s g s . g o v / f s / 2006 / 3135 /$ )

Anna, L.O., Pollastro, R.M., Gaswirth, S.B., Lewan, M.D., Lillis, P.G., Roberts, L.N.R., Schenk, C.J., Charpentier, R.R., Cook, T.A., and Klett, T.R., 2008, Assessment of undiscovered oil and gas resources of the Williston Basin Province of North Dakota, Montana, and South Dakota, 2008: U.S. Geological Survey Fact Sheet 2008-3092, 2 p. (Available at http://pubs.usgs.gov/fs/2008/3092/)

Brownfield, M.E., Charpentier, R.R., Cook, T.A., Klett, T.R., Pollastro, R.M., and Schenk, C.J., 2009, Assessment of undiscovered hydrocarbon resources of the Western Oregon and Washington Province: U.S. Geological Survey Fact Sheet 2009-3060, 2 p. (Available at http://pubs.usgs.gov/fs/2009/3060/) 
Brownfield, M.E., Tennyson, M.E., Ahlbrandt, T.S., Charpentier, R.R., Cook, T.A., Klett, T.R., Pollastro, R.M., and Schenk, C.J., 2006, Assessment of undiscovered gas resources of the Eastern Oregon and Washington Province, 2006: U.S. Geological Survey Fact Sheet 20063091, 2 p. (Available at http://pubs.usgs.gov/fs/2006/3091/)

Dubiel, R.F., Pitman, J.K., Pearson, O.N., Pearson, Krystal, Kinney, S.A., Lewan, M.D., Burke, Lauri, Biewick, L.R.H., Charpentier, R.R., Cook, T.A., Klett, T.R., Pollastro, R.M., and Schenk, C.J., 2012, Assessment of undiscovered oil and gas resources in conventional and continuous petroleum systems in the Upper Cretaceous Eagle Ford Group, U.S. Gulf Coast region, 2011: U.S. Geological Survey Fact Sheet 2012-3003, 2 p. (Available at http://pubs.usgs.gov/fs/2012/3003/)

Dubiel, R.F., Pitman, J.K., Pearson, O.N., Warwick, P.D., Karlsen, A.W., Coleman, J.L., Hackley, P.C., Hayba, D.O., Swanson, S.M., Charpentier, R.R., Cook, T.A., Klett, T.R., Pollastro, R.M., and Schenk, C.J., 2007, Assessment of undiscovered oil and gas resources in Tertiary strata of the Gulf Coast, 2007: U.S. Geological Survey Fact Sheet 2007-3066, 4 p. (Available at http://pubs.usgs.gov/fs/2007/3066/)

Dubiel, R.F., Warwick, P.D., Swanson, Sharon, Burke, Lauri, Biewick, L.R.H., Charpentier, R.R., Coleman, J.L., Cook, T.A., Dennen, Kris, Doolan, Colin, Enomoto, Catherine, Hackley, P.C., Karlsen, A.W., Klett, T.R., Kinney, S.A., Lewan, M.D., Merrill, Matt, Pearson, Krystal, Pearson, O.N., Pitman, J.K., Pollastro, R.M., Rowan, E.L., Schenk, C.J., and Valentine, Brett, 2011, Assessment of undiscovered oil and gas resources in Jurassic and Cretaceous strata of the Gulf Coast, 2010: U.S. Geological Survey Fact Sheet 2011-3020, 4 p. (Available at http://pubs.usgs.gov/fs/2011/3020/)

Dyman, T.S., Condon, S.M., Ahlbrandt, T.S., Charpentier, R.R., Cook, T.A., Klett, T.R., Lewan, M.D., Lillis, P.G., Pawlewicz, M.J., Pollastro, R.M., and Schenk, C.J., 2006, 2005 assessment of undiscovered oil and gas resources in Hanna, Laramie, Shirley Basins Province, Wyoming: U.S. Geological Survey Fact Sheet 2005-3125, 2 p. (Available at http://pubs.usgs.gov/fs/2005/3125/)

Flores, R.M., Anna, L.O., Dolton, G.L., Fox, J.E., French, C.D., Charpentier, R.R., Cook, T.A., Crovelli, R.A., Klett, T.R., Pollastro, R.M., and Schenk, C.J., 2002, Assessment of undiscovered oil and gas resources of the Powder River Basin Province of Wyoming and Montana, 2002: U.S. Geological Survey Fact Sheet 146-02, 2 p. (Available at http://pubs.usgs.gov/fs/fs-146-02/)

Hatch, J.R., Pawlewicz, M.J., Charpentier, R.R., Cook, T.A., Crovelli, R.A., Klett, T.R., Pollastro, R.M., and Schenk, C.J., 2003, Assessment of undiscovered oil and gas resources of the Black Warrior Basin Province, 2002: U.S. Geological Survey Fact Sheet 038-03, 2 p. (Available at http://pubs.usgs.gov/fs/fs-038-03/)

Higley, Debra, Charpentier, R.R., Cook, Troy, Klett, T.R., Pollastro, Richard, Schmoker, J.W., and Schenk, C.J., 2003, 2002 USGS assessment of oil and gas resource potential of the Denver 
Basin Province of Colorado, Kansas, Nebraska, South Dakota, and Wyoming: U.S. Geological Survey Fact Sheet 002-03, 4 p. (Available at $h t t p: / / p u b s . u s g s . g o v / f_{s} / f_{s}-002-03 /$ )

Higley, D.K., Cook, T.A., Pollastro, R.M., Charpentier, R.R., Klett, T.R., and Schenk, C.J., 2005, Assessment of undiscovered oil and gas resources of the Raton Basin-Sierra Grande Uplift Province of New Mexico and Colorado, 2004: U.S. Geological Survey Fact Sheet 20053027, 2 p. (Available at http://pubs.usgs.gov/fs/2005/3027/)

Higley, D.K., Gaswirth, S.B., Abbott, M.M., Charpentier, R.R., Cook, T.A., Ellis, G.S., Gianoutsos, N.J., Hatch, J.R., Klett, T.R., Nelson, Philip, Pawlewicz, M.J., Pearson, O.N., Pollastro, R.M., and Schenk, C.J., 2011, Assessment of undiscovered oil and gas resources of the Anadarko Basin Province of Oklahoma, Kansas, Texas, and Colorado, 2010: U.S. Geological Survey Fact Sheet 2011-3003, 2 p. (Available at http://pubs.usgs.gov/fs/2011/3003/)

Houseknecht, D.W., Coleman, J.L., Milici, R.C., Garrity, C.P., Rouse, W.A., Fulk, B.R., Paxton, S.T., Abbott, M.M., Mars, J.C., Cook, T.A., Schenk, C.J., Charpentier, R.R., Klett, T.R., Pollastro, R.M., and Ellis, G.S., 2010, Assessment of undiscovered natural gas resources of the Arkoma Basin Province and geologically related areas: U.S. Geological Survey Fact Sheet 2010-3043, 4 p. (Available at http://pubs.usgs.gov/fs/2010/3043/)

Kirschbaum, M.A., Anna, Larry, Collett, T.S., Cook, Troy, Dubiel, R.F., Finn, T.M., Hettinger, R.D., Henry, Mitchell, Johnson, E.A., Johnson, R.C., Lillis, P.G., Nelson, P.H., Nuccio, V.F., Rice, C.A., Roberts, L.N.R., and Roberts, S.B., 2002, Assessment of undiscovered oil and gas resources of the Uinta-Piceance Province of Colorado and Utah, 2002: U.S. Geological Survey Fact Sheet 026-02, 2 p. (Available at http://pubs.usgs.gov/fs/fs-0026-02/)

Kirschbaum, M.A., Anna, Larry, Collett, T.S., Cook, Troy, Dubiel, R.F., Finn, T.M., Hettinger, R.D., Henry, Mitchell, Johnson, E.A., Johnson, R.C., Lillis, P.G., Nelson, P.H., Nuccio, V.F., Rice, C.A., Roberts, L.N.R., and Roberts, S.B., 2003, Assessment of undiscovered oil and gas resources of the Uinta-Piceance Province of Colorado and Utah, 2002: U.S. Geological Survey Fact Sheet 157-02, 2 p. (Available at http://pubs.usgs.gov/fs/fs-157-02/)

Kirschbaum, M.A., Charpentier, R.R., Crovelli, R.A., Klett, T.R., Pollastro, R.M., and Schenk, C.J., 2004, Assessment of undiscovered oil and gas resources of the Wyoming Thrust Belt Province, 2003: U.S. Geological Survey Fact Sheet 2004-3025, 2 p. (Available at http://pubs.usgs.gov/fs/2004/3025/)

Kirschbaum, M.A., Condon, S.M., Finn, T.M., Johnson, R.C., Lillis, P.G., Nelson, P.H., Roberts, L.N.R., Roberts, S.B., Charpentier, R.R., Cook, Troy, Klett, T.R., Pollastro, R.M., and Schenk, C.J., 2008, Assessment of undiscovered oil and gas resources of the Bighorn Basin Province, Wyoming and Montana, 2008: U.S. Geological Survey Fact Sheet 2008-3050, 2 p. (Available at http://pubs.usgs.gov/fs/2008/3050/)

Kirschbaum, Mark, Finn, T.M., Hettinger, R.D., Johnson, E.A., Johnson, R.C., Kibler, Joyce, Lillis, P.G., Nelson, P.H., Roberts, L.N.R., Roberts, S.B., Charpentier, R.R., Cook, T.A., 
Crovelli, R.A., Klett, T.R., Pollastro, R.M., and Schenk, C.J., 2002, Assessment of undiscovered oil and gas resources of the Southwestern Wyoming Province, 2002: U.S. Geological Survey Fact Sheet 145-02, 2 p. (Available at http://pubs.usgs.gov/fs/fs-145-02/)

Kirschbaum, M.A., Finn, T.M., Johnson, R.C., Kibler, Joyce, Lillis, P.G., Nelson, P.H., Roberts, L.N.R., Roberts, S.B., Charpentier, R.R., Cook, Troy, Klett, T.R., Pollastro, R.M., and Schenk, C.J., 2005, Assessment of undiscovered oil and gas resources of the Wind River Basin Province, 2005: U.S. Geological Survey Fact Sheet 2005-3141, 2 p. (Available at http://pubs.usgs.gov/fs/2005/3141/)

Milici, R.C., and Hatch, J.R., 2004, Assessment of undiscovered Carboniferous coal-bed gas resources of the Appalachian Basin and Black Warrior Basin Provinces, 2002: U.S. Geological Survey Fact Sheet 2004-3092, 2 p. (Available at $h t t p: / / p u b s . u s g s . g o v / f s / 2004 / 3092 /)$

Milici, R.C., Ryder, R.T., Swezey, C.S., Charpentier, R.R., Cook, T.A., Crovelli, R.A., Klett, T.R., Pollastro, R.M., and Schenk, C.J., 2003, Assessment of undiscovered oil and gas resources of the Appalachian Basin Province, 2002: U.S. Geological Survey Fact Sheet 00903, 2 p. (Available at $h t t p: / / p u b s . u s g s . g o v / f s / f s-009-03 /$ )

Pearson, Krystal, Dubiel, R.F., Pearson, O.N., Pitman, J.K., Charpentier, R.R., Cook, T.A., Klett, T.R., Pollastro, R.M., and Schenk, C.J., 2011, Assessment of undiscovered oil and gas resources of the Upper Cretaceous Austin Chalk and Tokio and Eutaw Formations, Gulf Coast, 2010: U.S. Geological Survey Fact Sheet 2011-3046, 2 p. (Available at http://pubs.usgs.gov/fs/2011/3046/)

Pollastro, R.M., Cook, T.A., Roberts, L.N.R., Schenk, C.J., Lewan, M.D., Anna, L.O., Gaswirth, S.B., Lillis, P.G., Klett, T.R., and Charpentier, R.R., 2008: Assessment of undiscovered oil resources in the Devonian-Mississippian Bakken Formation, Williston Basin Province, Montana and North Dakota, 2008: U.S. Geological Survey Fact Sheet 2008-3021, 2 p. (Available at $h t t p: / / p u b s . u s g s . g o v / f s / 2008 / 3021 /$ )

Pollastro, R.M., Hill, R.J., Ahlbrandt, T.A., Charpentier, R.R., Cook, T.A., Klett, T.R., Henry, M.E., and Schenk, C.J., 2004, Assessment of undiscovered oil and gas resources of the Bend Arch-Fort Worth Basin Province of north-central Texas and southwestern Oklahoma, 2003: U.S. Geological Survey Fact Sheet 2004-3022, 2 p. (Available at http://pubs.usgs.gov/fs/2004/3022/)

Ridgley, J.L., Anna, L.O., Condon, S.M., Fishman, N.S., Hester, T.C., Lillis, P.G., Rowan, E.L., Charpentier, R.R., Cook, T.A., Crovelli, R.A., Klett, T.R., and Schenk, C.J., 2008, Assessment of undiscovered biogenic gas resources, North-Central Montana Province: U.S. Geological Survey Fact Sheet 2008-3036, 2 p. (Available at $h t t p: / / p u b s . u s g s . g o v / f s / 2008 / 3036 /$ )

Ridgley, J.L., Condon, S.M., Dubiel, R.F., Charpentier, R.R., Cook, T.A., Crovelli, R.A., Klett, T.R., Pollastro, R.M., and Schenk, C.J., 2002, Assessment of undiscovered oil and gas resources of the San Juan Basin Province of New Mexico and Colorado, 2002: U.S. Geological Survey Fact Sheet 147-02, 2 p. (Available at $h t t p: / / p u b s . u s g s . g o v / f s / f s-147-02 /)$ 
Roberts, Steve, Barker, C.E., Bird, K.J., Charpentier, R.R., Cook, Troy, Houseknecht, D.W., Klett, T.R., Pollastro, R.M.; and Schenk, C.J., 2006, Assessment of coalbed gas resources in Cretaceous and Tertiary rocks on the North Slope, Alaska, 2006: U.S. Geological Survey Fact Sheet 2006-3105, 2 p. (Available at http://pubs.usgs.gov/fs/2006/3105/)

Schenk, C.J., Charpentier, R.R., Cook, T.A., Dyman, T.S., French, C.D., Henry, M.E., Klett, T.R., Perry, W.J., Pollastro, R.M., and Potter, C.J., 2002, Assessment of undiscovered oil and gas resources of the Montana Thrust Belt Province, 2002: U.S. Geological Survey Fact Sheet 148-02, 2 p. (Available at http://pubs.usgs.gov/fs/fs-148-02/)

Schenk, C.J., Pollastro, R.M., Cook, T.A., Pawlewicz, M.J., Klett, T.R., Charpentier, R.R., and Cook, H.E., 2008, Assessment of undiscovered oil and gas resources of the Permian Basin Province of west Texas and southeast New Mexico, 2007: U.S. Geological Survey Fact Sheet 2007-3115, 4 p. (Available at http://pubs.usgs.gov/fs/2007/3115/)

Stanley, R.G., Charpentier, R.R., Cook, T.A., Houseknecht, D.W., Klett, T.R., Lewis, K.A., Lillis, P.G., Nelson, P.H., Phillips, J.D., Pollastro, R.M., Potter, C.J., Rouse, W.A., Saltus, R.W., Schenk, C.J., Shah, A.K., and Valin, Z.C., 2011, Assessment of undiscovered oil and gas resources of the Cook Inlet region, south-central Alaska, 2011: U.S. Geological Survey Fact Sheet 2011-3068, 2 p. (Available at http://pubs.usgs.gov/fs/2011/3068/)

Swezey, C.S., Hatch, J.R., Brennan, S.T., East, J.A., Rowan, E.L., Repetski, J.E., Charpentier, R.R., Cook, T.A., Klett, T.R., Pollastro, R.M., and Schenk, C.J., 2007, Assessment of undiscovered oil and gas resources of the Illinois Basin, 2007: U.S. Geological Survey Fact Sheet 2007-3058, 2 p. (Available at http://pubs.usgs.gov/fs/2007/3058/)

Swezey, C.S., Hatch, J.R., Hayba, D.O., Repetski, J.E., Charpentier, R.R., Cook, T.A., Klett, T.R., Pollastro, R.M., and Schenk, C.J., 2005, Assessment of undiscovered oil and gas resources of the U.S. portion of the Michigan Basin, 2004: U.S. Geological Survey Fact Sheet 2005-3070, 2 p. (Available at http://pubs.usgs.gov/fs/2005/3070/)

Warwick, P.D., Charpentier, R.R., Cook, T.A., Klett, T.R., Pollastro, R.M., and Schenk, C.J., 2007, Assessment of undiscovered oil and gas resources in Cretaceous-Tertiary coal beds of the Gulf Coast Region, 2007: U.S. Geological Survey Fact Sheet 2007-3039, 2 p. (Available at http://pubs.usgs.gov/fs/2007/3039/) 